\title{
Chitotriosidase Enzyme Activity and MiRNA-146a Expression and Their Value as Potential Biomarkers of Subclinical Atherosclerosis in Type 2 Diabetes Mellitus
}

\author{
Hala Mourad Demerdash ${ }^{1 *}$, Radwa Mohamed EISharaby ${ }^{2}$ and Yasser Mohamed Abdel Raouf ${ }^{3}$ \\ ${ }^{1}$ Clinical Pathology Department, Faculty of Pharmacy, Alexandria University Hospitals, Pharos University in Alexandria, Egypt \\ ${ }^{2}$ Clinical Pathology Department, Faculty of Medicine, Tanta University. Egypt \\ ${ }^{3}$ Department of Internal Medicine, Faculty of Medicine Tanta University, Egypt
}

\begin{abstract}
Background: Type 2 diabetes T2DM is a common disease with increased mortality and morbidity due to vascular complications. Carotid Intimal-Medial Thickness (CIMT) is being used as a marker to assess subclinical atherosclerosis.MicroRNA (miR-146a) is a new discoveredbiomarker that regulates endothelial cell function and vascular inflammation, together with Chitotriosidase (CHIT1)enzyme being involved mainly in the immune and inflammatory responses.

This study aimed to investigate an assumed association between CHIT1 activity and miR-146a gene expression in type 2 diabetes patients for detection of endothelial dysfunction and subclinical atherosclerosis, in association with CIMT.

Material and Methods: 30 control subjects (group I) and 100 patients diagnosed as T2DMclassified according to CIMT results; group II (38 patient) with CIMT value less than $0.7 \mathrm{~mm}$ and group III (62patients) with CIMT value more than $0.7 \mathrm{~mm}$. Plasma CHIT1 activity was measured fluorometricallyand quantitative Real Time PCR (qPCR) for miR-146a. Routine laboratory parameters such as blood glucose, lipid profile, glycated hemoglobin were also measured.
\end{abstract}

Results: revealedCHIT1 activity was significant high $(\mathrm{p}<0.001)$ in group II and III diabetic patients compared to group I, also it waspositively correlated with CIMT and other parameters as glycemic control, lipid profile, duration of disease and blood pressure. On the other hand, plasma miR-146a was significantly reduced $(\mathrm{p}<0.001)$ in group III and was negatively correlated with CIMT and other parameters.

Conclusion: IncreasedCHITlactivity may be due to the associated changes in the relative gene expression of miRNA 146a in patients with increased CIMT above $1 \mathrm{~mm}$. Moreover, evaluation of microRNA 146a gene expression can be used as useful biomarker for detection of endothelial dysfunction and development of atherosclerosis in T2DM.

Keywords: atherosclerosis; Chitotriosidase; MicroRNAs; Carotid Intimal-Medial Thickness.

Abbreviations: T2DM,Type 2 diabetes; ED,Endothelial dysfunction; CHIT1, enzyme chitotriosidase; CIMT, carotid intima-media thickness; HbA1C, glycated hemoglobin

\section{Introduction}

Type 2 diabetes (T2DM) is a chronic metabolic disease, thatresults from insulin resistance and or deficiency. It is associated with many metabolic abnormalities, such as obesity, hypertension, and dyslipidemia. However, pathologic hallmark of T2DM involves the vascular complications due to long term hyperglycemia ${ }^{[1]}$. Endothelial dysfunction (ED)contributes to the pathological progression ofvascular complications in T2DM.ED occurs as consequence of combination of several elements including genetic predisposition, systemic inflammation and cardiovascular risk factors ${ }^{[2]}$.

The endothelium is a key regulator of vascular function, and ED tends to be the initial event in macrovascular complications such as atherosclerosis inT2DM. Mechanisms of ED include the down-regulation of endothelial nitric oxide synthase levels, oxidative stress, activation of inflammatory pathways and differential expression of vascular endothelial growth factor and other inflammatory mediators [3]. A large body of evidence designates that inflammation is important in initiation of atherogenesis and development of subclinical atherosclerosis, manifested by increased carotid artery intima-media thickness and increased number of carotid plaques, in patients with T2DM ${ }^{[4]}$.

The enzyme chitotriosidase (CHIT1) belongs to the glycoside hydrolase family 18 . It is one of the most abundant and indicative proteins secreted by activated macrophages and neutrophil-specific granules. It has an active role in the immune response, being implicated in the activation and polarization of macrophages, propagating 
chronic inflammation, as well as activation of other immune cells such as T helper cells ${ }^{[5]}$.

MiRNAs are a group of approximately 20 to 22 nucleotides noncoding RNAs and have been proven to regulate gene expression at the post-transcriptional level. In addition, they can directly stimulate or inhibit target gene transcription by directly binding to promoter regions, RNAactivation phenomena. miR-146a is involved in modulation of the inflammatory pathways in T2DM ${ }^{[6]}$

Measurement of the carotid intima-media thickness (CIMT) has been recognized as a powerful method for identifying subclinical atherosclerosis, and increased thickness of the intima- media complex reflects local generalized atherosclerosis ${ }^{[7]}$. The objective of this study was to evaluate respective values of miRNA-146a and CHITlandtheir association with CIMT, being a valid marker of atherosclerosis in T2DM patients.

\section{Materials and Methods}

This cross-sectional study was carried out on 30 control subjects and 100 T2DM patients. Subject's selection was undertaken in collaboration between departments of Internal Medicine and Radiology, Tanta University Hospitals. The research protocol was performed in accordance Declaration of Helsinki and approved by the Medical Ethics Committee of the Faculty of medicine, Tanta University, from April 2015until April 2016. Informed written consent was obtained from all subjects included in the study.

Inclusion criteria were diabetes type 2 patients on diabetes therapy including insulin and/or oral hypoglycemic drugs more than five years. Exclusion criteria included type 1 diabetes, patients with history of familial dyslipidemia, hypothyroidism, liver or kidney disease, Cushing's syndrome, myocardial infarction during the last 6 months.

After an overnight fasting, $9 \mathrm{ml}$ blood was taken from every investigated subject. $4 \mathrm{ml}$ of EDTA whole blood, centrifuged at $4000 \mathrm{rpm}$ for $10 \mathrm{~min}$ at $4{ }^{\circ} \mathrm{C}$, plasma was separated and stored at $-80{ }^{\circ} \mathrm{C}$ for estimation of plasmachitotriosidase enzyme activity (CHIT1) andmiR- 146a gene expression. $2 \mathrm{ml}$ of EDTAwholeblood for Glycated Hemoglobin ( $\mathrm{Hb}$ A1C) was determined directly (without measurement of total hemoglobin) by turbidimetric inhibition immunoassay (Roche, Basel, Switzerland). $3 \mathrm{ml}$ of blood in a plain sterile tube and centrifuged at $4000 \mathrm{rpm}$ for $10 \mathrm{~min}$ at $4{ }^{\circ} \mathrm{C}$; serum was separated Fasting blood glucose (FBG) and the rest of sample stored at $-20^{\circ} \mathrm{C}$ for estimation of lipid profile. Also, $2 \mathrm{ml}$ of blood were collected in a plain sterile tube after twohours for post-prandial blood glucose (PPBG) determination.
Both FBG and PPBG were estimated by a glucose analyzer 2 (Beckman Instruments, Fullerton, CA) based on the glucose oxidase method.Lipid profile; Serum total cholesterol (T.C), serum triglyceride, high density cholesterol (HDL-C) and low density cholesterol (LDL-C) were determined with kits from SENTINEL CH. (via principle Eugenio 5-20155 MILAN-ITALY).

Plasma Chitotriosidase activity(CHIT1):was measured fluorimetricallyby enzymatic hydrolysis of the fluorimetric substrate 4-methylumbelliferyl- $\beta-D-N N, N$ 'triacetylchitotriose (Sigma-Aldrich ChemieGmBH, Taufkirchen, Germany) and release of the fluorescent molecule 4-methylumbelliferone (4MU) which was read in a Microfluor 2 ® plate by a fluorimeter (BIO-TEK 5SynergyHT; Biotek Instruments Inc., Winooski, VT).

Plasma miR-146a gene expression quantification by real-time PCR (RTq-PCR): Extraction of miRNA by miRNeasyMiniKit (supplied by Qiagen, Valencia, CA, USA) Reverse transcription was performed for cDNA synthesis using the miRNeasy plasma Reverse Transcription Kit (Qiagen, Valencia, CA, USA) according to the manufacturer's instructions.

The expression miR-146a was evaluated by qRTPCR analysis according to the manufacturer's protocol. miR-146a Forward primer:5'TGAGAACTGAATTCCATGGGTT-3' while miR-146aReverse primer:5'-ATCTACTCTCTCCAGGTCCTCA-3. Reference gene U6 small nuclear RNA (snRNA) primers, Forward primer:5'-CTCGCTTCGGCAGCACA-3', while Reverse primer:5'-AA CGCTTCACGAATTTGCGT-3'.

For real-time PCR, the cDNA template was mixed with SYBER Green Master Mix (Qiagen, Valencia, CA, USA). PCR array plate enriched with forward and reverse miRNAspecific primers. Real-time PCR reactions were performed using an Applied Biosystems 7300HT analyzer.The cycle threshold (CT) is defined as the number of cycles required for the fluorescent signal to cross the threshold in realtime PCR. The quantification of miRNAs gene expression was reported as the $\Delta \mathrm{CT}$ value, which was calculated by subtracting the CT values of miRNA Reference gene from the $\mathrm{CT}$ values of the target miRNAs as following:

The comparative miR-146a Expression as Measured by: $\left(\Delta \Delta \mathrm{C}_{\mathrm{t}}\right)=\left(\mathrm{C}_{\mathrm{t}}\right.$ miR-146a normal - $\mathrm{C}_{\mathrm{t}} \mathrm{RNU6}$ normal $)-\left(\mathrm{C}_{\mathrm{t}}\right.$ miR-146a RA- $C_{t}$ RNU6 RA). The Relative expression in the target gene was calculated based onthe estimated mean difference (2)- $\Delta \Delta \mathrm{Ct}$

Measurement of Carotid Intima-Media Thickness (CIMT): CIMT was determined using a standardized protocol; patient was examined in supine position with 
head turned $45^{\circ}$ opposite to the side being tested. CIMT measurement was done using high resolution B-mode (9MHz) ultrasound (Toshiba SSA370A, Japan). The CIMT was defined as distance between edges of the lumen-intima and the media-adventitia echos, in a plaquefree section. CIMT measured bilaterally, CIMT values $\leq 0.7 \mathrm{~mm}$ indicated normal CIMT and values $>0.9 \mathrm{~mm}$ indicated increased CIMT[8]. All measurements were performed blinded for patients' clinical data by the same experienced sonographer.

Statistical Analysis: Data were fed to the computer and analyzed using IBM SPSS software package version 20.0. Qualitative data were described using number and percent. Quantitative data were described using range (minimum and maximum), mean, standard deviation and median. Significance of the obtained results was judged at the $5 \%$ level.

\section{The used tests were}

1- ONE WAY: Analysis of Variance (ANOVA), was performed for comparison between more than two sample

2- Correlation coefficient; to determine the association between each two studied variables.

\section{RESULTS}

Studied subjects were classified into three groups; group I were 30 healthy control subjects; diabetic patients were divided into groups based on results of CIMT into 2 groups; group IIincluded 38 T2DM patients(with CIMT values less than $0.7 \mathrm{~mm}$ )none of them had history of transient ischemic attacks or ischemic stroke, while group III included 62 T2DM patients (with CIMT values of $0.9 \mathrm{~mm}$ or more). The disease duration was significantly higher in group III (11.43 \pm 3.1 years) compared to group II ( $7.8 \pm 2.25$ years). Studied groups revealed no significant difference as regard age; in which group, I was $44.87 \pm$ 6.03 years, group II was $43.92 \pm 3.94$ yearsand group III was $44.22 \pm 3.66 y e a r s$. Also, male to female ratio in group I was $1.5: 1(18: 12)$, in group II $2.2: 1.0$, (26:12) while in group III $1.6: 1.0(38: 24)$ there was no statistically significant difference between the males to females ratio in studied groups.Table (1) shows patientsbiochemical data and blood pressure of studied groups.

The carotid intima thickness (CIMT) was significantly higher in group III compared to both group II and I(table 2). There was a significant increase in CHIT1 enzyme activity in group II and III compared to group I. The level of miR-146a gene expression was significantly increased in group III more than group I and II(table 3). Correlation between CIMT values and studied biomarkers was performed; results revealed a positive correlation between CIMT and CHIT1 and negative correlation with miR-146a gene expression (table 4).

The significant parameters which had an effect on CIMT were duration of diabetes CHIT1 enzyme activity and miR-146a gene expression and HbA1C level (table 5).

Table 1: Blood pressure and Biochemical data of the three studied groups

\begin{tabular}{|l|c|c|c|c|c|}
\hline & $\begin{array}{c}\text { Group I } \\
(\mathrm{N}=30)\end{array}$ & $\begin{array}{c}\text { Group II } \\
(\mathrm{N}=38)\end{array}$ & $\begin{array}{c}\text { Group III } \\
(\mathrm{N}=62)\end{array}$ & F & F \\
\hline FBG $(\mathrm{mg} / \mathrm{dl})$ & $87.9 \pm 3.9^{\mathrm{a}}$ & $129.31 \pm 11.8^{\mathrm{b}}$ & $149.54 \pm 21.44^{\mathrm{c}}$ & 22.3 & $0.001^{*}$ \\
\hline PPBG $(\mathrm{mg} / \mathrm{dl})$ & $112.67 \pm 7.67^{\mathrm{a}}$ & $193.61 \pm 45.23^{\mathrm{b}}$ & $226.77 \pm 65.9^{\mathrm{c}}$ & 22.6 & $0.001^{*}$ \\
\hline Hb A1C & $6.41 \pm 1.42^{\mathrm{a}}$ & $7.94 \pm 1.96^{\mathrm{ab}}$ & $8.62 \pm 2.1^{\mathrm{b}}$ & 8.22 & $0.013^{*}$ \\
\hline Systolic BP $(\mathrm{mm} \mathrm{Hg})$ & $114.1 \pm 5.89^{\mathrm{a}}$ & $123.7 \pm 14.6^{\mathrm{b}}$ & $131.2 \pm 15.7^{\mathrm{b}}$ & 15.6 & $0.0052^{*}$ \\
\hline Diastolic BP $(\mathrm{mm} \mathrm{Hg})$ & $76.4 \pm 4.2$ & $85.1 \pm 7.8$ & $89.8 \pm 6.4$ & 2.65 & 0.122 \\
\hline T.C $(\mathrm{mg} / \mathrm{dl})$ & $153.50 \pm 15.08^{\mathrm{a}}$ & $225.46 \pm 3.16^{\mathrm{b}}$ & $235.82 \pm 9.05^{\mathrm{b}}$ & 62.25 & $0.0001^{*}$ \\
\hline T.G $(\mathrm{mg} / \mathrm{dl})$ & $107.62 \pm 14.94^{\mathrm{a}}$ & $140.95 \pm 16.40^{\mathrm{b}}$ & $181.80 \pm 8.01^{\mathrm{c}}$ & 44.98 & $0.0001^{*}$ \\
\hline HDL-C $(\mathrm{mg} / \mathrm{dl})$ & $49.57 \pm 4.13^{\mathrm{a}}$ & $40.95 \pm 4.43^{\mathrm{b}}$ & $43.71 \pm 6.26^{\mathrm{ab}}$ & 8.2 & $0.0026^{*}$ \\
\hline LDL-C $(\mathrm{mg} / \mathrm{dl})$ & $84.87 \pm 8.15^{\mathrm{a}}$ & $129.32 \pm 6.13^{\mathrm{b}}$ & $138.50 \pm 7.76^{\mathrm{b}}$ & 74.2 & $0.0001^{*}$ \\
\hline
\end{tabular}

* Statistically significant at $(P \leq 0.05)$

Data are expressed as mean $\pm S D ; p<0.05$ was considered statistically significant.

Table 2: Carotid intima thickness (CIMT) in the three studied groups.

\begin{tabular}{|c|c|c|c|}
\hline & Group I & Group II & Group III \\
\hline CIMT $(\mathrm{mm})$ & $0.60 \pm 0.07$ & $0.76 \pm 0.048$ & $1.14 \pm 0.09$ \\
\hline P & & 22.6 & \\
\hline
\end{tabular}

* Statistically significant at $(P \leq 0.05)$ 
Table 3: biomarkers of endothelial dysfunction (ED) in the three studied groups.

\begin{tabular}{|c|c|c|c|c|c|}
\hline & Group I & Group II & Group III & F & P \\
\hline CHIT1 enzyme activity & $193.647 \pm 17.40^{\mathrm{a}}$ & $313.78 \pm 8.65^{\mathrm{b}}$ & $335.85 \pm 20.29^{\mathrm{b}}$ & 49.8 & $0.0001^{*}$ \\
\hline miR-146a & $0.93 \pm 0.12 \mathrm{a}$ & $4.36 \pm 0.77_{\mathrm{b}}$ & $0.79 \pm 0.02 \mathrm{c}$ & 32.6 & $0.001^{*}$ \\
\hline
\end{tabular}

* Statistically significant at $(P \leq 0.05)$.

Table 4: Correlation between CIMT and Endothelia dysfunction biomarkers.

\begin{tabular}{|c|c|c|c|c|}
\hline \multirow{2}{*}{ CIMT } & \multicolumn{2}{|c|}{ Group II } & \multicolumn{2}{c|}{ Group III } \\
\cline { 2 - 5 } & $\mathbf{r}$ & $\mathbf{p}$ & $\mathbf{~ r}$ \\
\hline CHIT1 enzyme activity & 0.801 & $0.001^{*}$ & 0.599 & $0.016^{*}$ \\
\hline miR-146a & -0.521 & $0.023^{*}$ & -0.562 & $0.011^{*}$ \\
\hline
\end{tabular}

* Statistically significant at $(P \leq 0.05)$.

Table (5): Multiple logistic regression analysis using different biologically important variables as independent variables, and CIMT as the dependent variable.

\begin{tabular}{|c|c|c|c|c|c|}
\hline \multirow{2}{*}{ Model } & \multicolumn{2}{|c|}{ Unstandardized Coefficients } & \multirow{2}{*}{$\begin{array}{c}\text { Standardized Coefficients } \\
\text { Beta }\end{array}$} & \multirow{2}{*}{$\mathbf{t}$} & \multirow{2}{*}{ Sig. } \\
\hline & B & Std. Error & & & \\
\hline Constant & -0.622 & .228 & & -3.21 & $.001^{*}$ \\
\hline age & -0.103 & .104 & $-.051-$ & .106 & .32 \\
\hline Duration of disease & -0.063 & .033 & .065 & 3.165 & $.026^{*}$ \\
\hline T.C. & -0.0013 & .006 & $-.015-$ & $-.046-$ & .961 \\
\hline TG & 0.0016 & .0001 & .025 & .442 & .626 \\
\hline HDL-C & 0.005 & .004 & .161 & 1.179 & .241 \\
\hline LDL-C & -0.0028 & .004 & .006 & .011 & 0.980 \\
\hline T.C./HDL & 0.013 & .100 & .086 & .135 & .893 \\
\hline LDL/HDL & 0.049 & .167 & .189 & .293 & .770 \\
\hline CHIT1 enzyme activity & 0.026 & .0017 & .825 & 7.11 & $.0001^{*}$ \\
\hline miR-146a & 0.052 & .006 & .323 & 4.003 & $.016^{*}$ \\
\hline $\mathrm{Hb}$ A1C & 0.107 & .201 & .425 & 3.25 & $.021^{*}$ \\
\hline
\end{tabular}

a. Dependent Variable: CIMT

\section{Discussion}

Type 2 diabetes (T2DM) is associated with increased morbidity and mortality due to vascular complications. Hyperglycemia results in low-grade inflammation and formation of advanced glycosylated end-products (AGE). Endothelial dysfunction as consequenceof chronic inflammation evokes premature onset of atherosclerosis in $\mathrm{T} 2 \mathrm{DM}^{[2]}$.

Pathogenesis of atherosclerosis necessitates comprehensive understanding of the proteins secreted in the vessel wall by macrophages and the molecular mechanisms that regulate gene expression that participate in this process.In this study, we observed a relationship between two biomarkers; CHIT1 enzyme activity and miR-146a gene expression in assessment of subclinical atherosclerosis in view of CIMT being an establishedindicator of cardiovascular risk.
Several studies reported CIMT values below $0.7 \mathrm{~mm}$ is considered normal, while CIMT of $1 \mathrm{~mm}$ or more is always associated with anenhanced absolute risk of Chronic Heart Disease ${ }^{[9,10]}$. Diabetic patients were classified according to CIMT value wherein CIMT $>0.9 \mathrm{~mm}$ was considered as increased CIMT (indicating vascular hypertrophy) [10]. In this study, results revealed that diabetic patients (group III) displayed significant increase in CIMT values compared to other groups; group I and II patients had CIMT values of $0.60 \pm 0.07$ and $0.76 \pm 0.048 \mathrm{~mm}$ respectively, while group III patients had CIMT value of $1.14 \pm 0.09 \mathrm{~mm}$. Raised CIMT values were explained by deposition of lipid-laden macrophages in the wall of blood vessel which promoted the expression of different genes that stimulated the inflammatory process in atherogenesis. This process consequently resulted in arterial stiffness and thickening of the intima and media 
of the arterial wall ${ }^{[1]}$. Group III patients had an extended duration of diabetes $11.43 \pm 2.1$ years and poor glycemic control as determined by HbA1C compared to group II patients where shorter disease duration was $7.8 \pm 2.25$ years and better glycemic control. These results came in concordance with Butt et al and Sibal et al.; they concluded upsurge of CIMT value was influenced by duration of disease and glycemic control $[9,10]$.

Results of present study revealed that total cholesterol and LDL-cholesterol were significantly increased in diabetic patients compared to control group with no significant difference between group II and III.Regression analysis was done and detected lack of relationship between CIMT and parameters of lipid metabolism in T2DM. Likewise, Byrkjeland et al found no correlation between in LDLcholesterol and CIMT, inferring that increase in LDLcholesterol did not influence CIMT progression ${ }^{[12]}$. In contrast, Kota et al reported that glycemic parameters as FBG, and $\mathrm{HbA} 1 \mathrm{C}$ collectively with lipid parameters were all significantly higher in diabetic patients with increased CIMT values ${ }^{[11]}$.

As regards Systolic BP it was significantly elevated in group II and III compared to group I, with no significant difference observed between group II and III.Similarly, Perumal et al reported that hypertension had a significant positive correlation with CIMT in T2DM ${ }^{[13]}$. Consequently, univariate analysis of present work discloses that hyperglycemia, raised systolic BP, increased T-cholesterol and LDL-cholesterol are all risk factors that predispose to the development of atherosclerosis, over prolonged period more than 5 years as perceived in group III.

Our results demonstrated significant increase in CHIT1 activity in group II and III compared to group I, with no significant difference between group II and III. Increased activity observed in T2DM patientshad a significant positive relationship with CIMT. It implied that CHIT activity may exhibit a key role in vascular inflammation, generation of Reactive oxygen species, and endothelial dysfunction, triggering the progression of atherosclerosis ${ }^{[14]}$. CHIT1 has transglycosidase activity, with resultant formation of chitotetraose while chitin being degraded; Chitotetraose acts as signaling molecule that plays an important regulatory role on the immune system ${ }^{[15]}$. In accordance, CHIT1 activity is increased in patients with atherosclerosis and is correlated with the severity of the disease ${ }^{[16,17]}$.

Furthermore, Artieda et al confirmed that CHIT1 is upregulated in response to inflammatory stimuli, and is responsible for macrophagesactivation and polarization. As macrophages shift toward M1-M2 phenotype; M1 phenotype, CHIT1 becomes implicated in phagocytosis, and induction of the adaptive immune response. While in M2 cells they promote phagocytosis of cellular debris, and coordinate tissue repair through the production of extracellular matrix proteins ${ }^{[18]}$. Also, Boot et al. established a relationship between increased CHIT1 activity and lipidladen macrophages in atherosclerotic vessel wall ${ }^{[19]}$.

In addition, CHIT1 is expressed during dendritic cell (DC) differentiation and maturation.DCs arelocalized in the intima of atherosclerotic vessels and they are activated by oxidized LDL-cholesterol (oxLDL) in atherosclerotic plaque. Also, intense expression of CHIT1 was identified in mature DCs indicating itsvital role in inflammation ${ }^{[20]}$.

CHIT1 is expressed in neutrophils upon stimulation with interferon (IFN) $\gamma$, tumor necrosis factor (TNF)- $\alpha{ }^{[16]}$. Żurawska-Płaksej et al reported involvement of neutrophilderived chitinasesas novel group of molecules, which may contribute to metabolic derangement and inflammatory pathways in the course of T2DM particularly vascular complications ${ }^{[21]}$. In this work, elevated CHIT1 activity in diabetic groups was coupled with poor glycemic control as evident by elevated FBG, PPBG levelsas well as HbA1C. In addition, prolonged duration of disease particularly in group III patients may suggest its participation in the course of diabetes. On the contrary, Ramanathan et al. proposed that CHIT1 is a major protein product of activated macrophages, and increase of CHIT1 activity may be generated by natural age-related chronic macrophage activation, not an inflammatory condition ${ }^{[22]}$.

Several miRNAs have emerged as possible regulators of endothelial cell function in diabetic microangiopathy; miR$146 \mathrm{a}$ is upregulated in endothelial cells upon stimulation by pro-inflammatory mediators, nuclear factor NF- $\mathrm{\kappa B}^{[23]}$. Yang et al found that miR-146a decreases lipid uptake and cytokine release in oxLDL-stimulated macrophages by inhibiting activation of toll-like receptor 4 signaling pathway, suggesting it may have a protective role against the development of atherosclerosis [24]

miR-146a gene expression was evaluated in this work; its level was significantly reduced in group III relative to both group II and I. In other words, its level was significantly decreased in group III patients with CIMT level above $1 \mathrm{~mm}$. Reduced miR-146a expression in group III is characteristically considered a warning of a proinflammatory condition. Moreover, results demonstrated an inverse relationship between miR-146a expression and CIMT, while positive relationship between CIMT and $\mathrm{Hb}$ A1C and CHIT1 activity. In agreement with our results, Balasubramanyam et al displayed an inverse relationship between miR-146a expression and glycated hemoglobin, 
tumor necrosis factor receptor-associated factor (TRAF)-6, and NF-kB mRNA levels and circulatory levels of TNF- $\alpha$ and IL-6 ${ }^{[25]}$. Also, Boldin et al supposed that targeted miR146a deletion in mice leads to unwarranted production of proinflammatory cytokines (TNF $\alpha$, IL-6) ${ }^{[26]}$.

In addition, Rong et al reported miR-146a overexpression is caused by increased plasma heme oxygenase-1 concentrations and other anti-oxidant enzymes [27]. Furthermore, downregulation of miR-146a in peripheral blood in diabetic patients in response to hyperglycemic, suggested that it may be implicated in impaired proangiogenic effect and disturbed cellular metabolic control, which could be underlying molecularmechanisms for T2DM pathogenesis ${ }^{[28,29]}$.

Accordingly, over expression of miR-146 in endothelial cells inhibits the inflammatory response prompted by IL-1 $\beta$ and on the contrary inhibition of miR-146a exacerbates the inflammatory response to IL-1 ${ }^{[28]}$. In addition, miR-146 atargets two serine/threonine kinases, interleukin-1 receptor-associated kinase 1 (IRAK1) and tumor necrosis factor (TNF) receptor-associated factor 6 (TRAF6), upon activation they become associated with the interleukin-1 receptor (IL-1R) and are therefore responsible for IL-1-induced upregulation of NF-kB. This binding results in suppression of NF- $\kappa B$ 's target genes; such as the interleukins IL-6, IL-8, IL-1 $\beta$, and

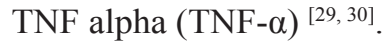

\section{Conclusion}

On the basis of these significant alterations in miR-146a and CHIT1 activity in group III patients with increased CIMT values, it is probable to conclude that as T2DM progresses; metabolic disturbances develop allowing for activation of the inflammatory response in those patients. Data obtained indicate potential role of CHIT1 enzyme in prediction and diagnosis of endothelial dysfunction and its increased activity may be due to the change in the relative gene expression of miR-146aas displayed by increased CIMT. However, further studies are needed to establish the diagnostic value (specificity and sensitivity) of both biomarkers in early detection of atherosclerosis in diabetic patients.

\section{Acknowledgements}

We kindly appreciate the support of Professor Dr Aml S Bendary (Prof of Clinical Pathology at Faculty of Medicine Tanta University) in revising it critically for important intellectual content.

\section{References}

1. Chawla A, Chawla R, Jaggi S. Microvasular and macrovascular complications in diabetes mellitus: Distinct or continuum? Indian J EndocrMetab 2016; 20:546-53.
2. Avogaro A, Albiero M, Menegazzo L, Kreutzenberg SD, Fadini GP. Endothelial dysfunction in diabetes - the role of reparatory mechanisms. Diabetes Care. 2011;34(2):285-90.

3. Brownlee M. Biochemistry and molecular cell biology of diabetic complications. Nature. 2001; 414:813-20.

4. Mudau M, Genis A, Lochner A, and Strijdom H. "Endothelial dysfunction: the early predictor of atherosclerosis". Cardiovascular Journal of Africa. 2012; 23 (4): 222-231.

5. Di Rosa M, Malaguarnera G, De Gregorio C, Drago F, and Malaguarner L. Evaluation of CHI3L-1 and CHIT-1 Expression in Differentiated and Polarized Macrophages. Inflammation. 2013; 36(2):482-492.

6. Sheetz MJ, King GL.Molecular understanding of hyperglycemia's adverse effects for diabetic complications. JAMA2002; 288: 2579-2588.

7. Nomura K, Hamamoto Y, Takahara S, Kikuchi O, Honjo S, Ikeda H, Wada Y, Nabe K, Okumra R, Koshiyama H: Relationship between carotid intima media thickness and silent cerebral infarction in Japanese subjects with type 2 diabetes. Diabetes Care 2010;33(1):168-70.

8. Gayathri R, Chandni R, Udayabhaskaran V. Carotid artery intima media thickness in relation with atherosclerotic risk factors in patients with type 2 diabetes mellitus. J Assoc Physicians India 2012;60:20-4.

9. Butt MU, Zakaria M. Association of common carotid intimal medial thickness (CCA-IMT) with risk factors of atherosclerosis in patients with type 2 diabetes mellitus. J Pak Med Assoc. 2009;59(9):590-593.

10. Sibal L, Agarwal SC, Home PD. Carotid intima-media thickness as a surrogate marker of cardiovascular disease in diabetes. Diabetes, Metabolic Syndrome and Obesity: Targets and Therapy. 2011; 4:23-34.

11. Kota S, Mahapatra G, and Mod K. Carotid intima media thickness in type 2 diabetes mellitus with ischemic stroke. Indian J of Endocrinol Metab.2013; 17(4): 716-722

12. Byrkjeland R, Stensæth K, Anderssen S, Njerve I, Arnesen $\mathrm{H}$, Seljeflot I, and Solheim S. Effects of exercise training on carotid intima-media thickness in patients with type 2 diabetes and coronary artery disease. Influence of carotid plaques.Cardiovasc Diabetol. 2016; 15: 13.

13. Perumal K, Santha K, SethupathyS, Sethurajan S, Mahendran K, BalasubramaniyanS.Plasma homocysteine level and carotid intima-media thickness in type 2 diabetic patients. International J of Med Science and Public Health. 2015; 4 (10):1404-1408.

14. Di Rosa $M$ and Malaguarnera L. Chitotriosidase: A New Inflammatory Marker in Diabetic Complications. Pathobiology 2016; 83:211-219.

15. Aguilera B, Ghauharali-van der Vlugt K, Helmond MT, Out JM, Donker-Koopman WE, Groener JE, et al: Transglycosidase activity of chitotriosidase: improved enzymatic assay for the human macrophage chitinase. J BiolChem 2003;278: 40911-40916. 
16. Karadag B, Kucur M, Isman FK, Hacibekiroglu M, Vural VA: Serumchitotriosidase activity in patients with coronary artery disease. Circ J2008, 72:71-75.

17. Kitamoto S, Egashira K, Ichiki T, Han X, McCurdy S, Sakuda S, Sunagawa K, andBoisvert W. Chitinase Inhibition Promotes Atherosclerosis in Hyperlipidemic Mice. The American Journal of Pathology.2013; 183(1):313-325.

18. Artieda M, Cenarro A, Ganan A, Lukic A, Moreno E, Puzo J, et al: Serum chitotriosidase activity, a marker of activated macrophages, predicts new cardiovascular events independently of C-reactive protein. Cardiology 2007; 108: 297-306.

19. Boot RG, van Achterberg TA, van Aken BE, Renkema GH, Jacobs MJ, Aerts JM, de Vries CJ. Strong induction of members of the chitinase family of proteins in atherosclerosis: chitotriosidase and human cartilage gp-39 expressed in lesion macrophages. ArteriosclerThrombVascBiol 1999; 19: 687-94.

20. Di Rosa M TD, Saccone S, Distefano G, Basile MS, Di Raimondo F, MalaguarneraL.Chitotriosidase expression during monocyte-derived dendritic cells differentiation and maturation. Inflammation 2015;38:2082-2091.

21. Żurawska-Płaksej E, Ługowska A, Hetmańczyk K, KnapikKordecka M, PiwowaA. Neutrophils as a source of chitinases and chitinase-like proteins in type 2 diabetes.PLoS One 2015; 10:e141730.

22. Ramanathan $R$, Kohli A, Ingaramo $M$, Jain A, Leng $S$, Punjabi P, Walston J and Fedarko N. Serum chitotriosidase, a putative marker of chronically activated macrophages, increases with normal aging. J Gerontol A Biol Sci Med Sci 2013; 68: 1303-10.
23. Siasos G, Kollia C, Tsigkou V, Basdra E, Lymperi M, Oikonomou E, Kokkou E, Korompelis P, Papavassiliou A. MicroRNAs: Novel diagnostic and prognostic biomarkers in atherosclerosis. Curr. Top. Med. Chem. 2013; 13:1503-1517.

24. Yang K, He Y, Wang Z, Lu L, Chen Q, Liu J, Sun Z, Shen W. MiR-146a inhibits oxidized low-density lipoprotein-induced lipid accumulation and inflammatory response via targeting toll-like receptor 4. FEBS Lett. 2011; 585:854-860.

25. Balasubramanyam M, Aravind S, Gokulakrishnan K, Prabu P, Sathishkumar C, Ranjani H, Mohan V. Impaired MIR-146a expression links subclinical inflammation and insulin resistance in type 2 diabetes. Mol. Cell Biochem. 2011;351:197-205.

26. Boldin M, Taganov K, Rao D, Yang L, Zhao J, Kalwani M, Garcia-Flores Y, Luong M, Devrekanli A, Xu J, Sun G, Tay J, Linsley P, Baltimore D. miR-146a is a significant brake on autoimmunity, myeloproliferation, and cancer in mice. J. Exp. Med.2011;208:1189-1201.

27. Rong Y, Bao W, Shan Z, Liu J, Yu X, Xia S, Gao H, Wang X, Yao P, Hu F, Liu L.Increased MicroRNA-146a Levels in Plasma of Patients with Newly Diagnosed Type 2 Diabetes Mellitus. PLoS ONE.2013; 8(9): e73272.

28. O'Connell RM, Rao DS, Baltimore D. MicroRNA regulation of inflammatory responses. Annu Rev Immunol 2012 30:295-312.

29. Li K, Ching D, Luk FS, Raffai RL. Apolipoprotein E enhances microRNA-146a in monocytes and macrophages to suppress nuclear factor- $\mathrm{kB}$-driven inflammation and atherosclerosis. Circ Res. 2015;117: e1-e11.

30. Fernandez-Valverde SL, Taft RJ, Mattick JS. MicroRNAs in beta- cell biology, insulin resistance, diabetes and its complications. Diabetes. 2011; 60: 1825-1831.

*Corresponding author:

Hala Demerdash, 75 Ismail Serry Street, Smouha-Sidi Gaber District-Zip code 21523, Alexandria, Egypt.

Phone: +2034258618, +201229501257

Email: demerdashh@yahoo.com

Date of Submission : 10.02.2017

Date of Acceptance : 01.04.2017

Financial or other Competing Interests: None.

Date of Publication : 04.07.2017 